\title{
AVALIAÇÃO FÍSICO-QUÍMICA DA INFLUÊNCIA DA COBERTURA BIODEGRADÁVEL DE GELATINA COM ADIÇÃO DE ÓLEO ESSENCIAL DE ALECRIM EM CORTES DE LOMBO SUÍNO
}

\author{
PHYSICAL-CHEMICAL EVALUATION OF THE INFLUENCE OF \\ BIODEGRADABLE COVERAGE OF GELATIN WITH ADDITION OF ESSENTIAL \\ OIL OF ALECRIM IN PORK LOIN CUTS
}

\author{
D. R. P. MARQUES ${ }^{1}$; A. L. S. MIRANDA ${ }^{1}$; G. P. CARDOSO ${ }^{2}$; I. C. GUIMARÃES ${ }^{1}$ \\ Universidade Federal de Viçosa, Campus Rio Paranaíba \\ ${ }^{2}$ Universidade dos Vales do Jequitinhonha e Mucuri - Campus JK \\ E-mail: david.paixao@ufv.br
}

\author{
$\underline{\operatorname{article~info}}$ \\ Article history: \\ Received 12 May 2017 \\ Accepted 3 August 2017 \\ Available online 20 September 2017
}

PALAVRAS-CHAVE:Revestimento; Óleo essencial; Rosmarinus officinalis; Carne suína.

RESUMO: Este trabalho teve como objetivo avaliar a influência do revestimento a base de gelatina adicionado de óleo essencial de alecrim nas características físico-químicas de cortes de lombo suíno durante 8 dias de armazenamento refrigerado. As amostras passaram por análises de $\mathrm{pH}$, umidade, cinzas, perda de peso e firmeza. Os resultados mostraram que as análises de umidade, cinzas, perda de peso e firmeza não foram afetadas significativamente pela interação Tipo de revestimento x tempo de armazenamento, sendo pH a única análise que foi afetado pela interação. $\mathrm{O}$ pH encontrado ao final dos 8 dias de armazenamento caracterizou todas as amostras como inicialmente degradados e a análise de perda de peso mostrou que os tratamentos que continham revestimento são eficazes para minimizar esta perda, visto que apresentaram menores valores para estas análises.

\begin{abstract}
The objective of this work was to evaluate the influence of the gelatin-based coating added with essential rosemary oil on the physical-chemical characteristics of pork loin cuts during 8 days of refrigerated storage. The samples were analyzed for $\mathrm{pH}$, moisture, ash, weight loss and firmness. The results showed that the moisture, ash, weight loss and firmness analyzes were not significantly affected by the interaction type of coating $x$ storage time, $p H$ being the only analysis that was affected by the interaction. The $p H$ found at the end of the 8 days of storage characterized all the samples as initially degraded and the weight loss analysis showed that the treatments that contained coating were effective to minimize this loss, since they presented lower values for these analyzes.
\end{abstract}

\section{INTRODUÇÃO}

A carne suína é uma das fontes de proteína animal mais importantes no mundo, representando quase metade do consumo e da produção de carnes, sendo que a maior 


\section{The Journal of Engineering and Exact Sciences - \\ JCEC \\ ISSN: 2527-1075}

produção ocorre na China, seguida dos Estados Unidos (FAO, 2016). Estudos e investimentos na suinocultura posicionaram o Brasil em quarto lugar no ranking de produção e exportação mundial de carne suína. Alguns elementos como sanidade, nutrição, bom manejo da granja, produção integrada e, principalmente, aprimoramento gerencial dos produtores, contribuíram para aumentar a oferta interna e colocar o País em destaque. O Brasil representa $10 \%$ do volume exportado de carne suína no mundo, chegando a lucrar mais de US\$ 1 bilhão por ano. Esses fatores apontam para um crescimento ainda mais satisfatório. Em relação às exportações, a representatividade do mercado brasileiro de carne suína saltará de $10,1 \%$, em 2008, para 21\% em 2018/2019 (MAPA, 2016).

De acordo com Sarcinelli et al. (2007), sua qualidade deve apresentar características sensoriais (aparência, cor, sabor, textura e suculência), conteúdo de nutrientes (proteína, pigmento e gordura intramuscular, principalmente), aspectos higiênicos e sanitários e a capacidade da carne em reter fluído durante a manipulação e processamento. Se algumas dessas características estiverem alteradas, a qualidade estará comprometida, resultando em perdas econômicas para a indústria da carne e que são repassadas para o consumidor.

A cobertura comestível biodegradável é uma película fina à base de material biológico, que pode agir como uma barreira a elementos externos tais como umidade, óleo e gases e, consequentemente, confere maior proteção ao produto revestido, aumentando assim seu armazenamento. Entre as propriedades funcionais dessas coberturas podem ainda ser mencionados o transporte de gases $(\mathrm{O} 2$ e $\mathrm{CO} 2)$ e de solutos; a retenção de compostos aromáticos e o transporte e a incorporação de aditivos alimentícios, tais como, nutrientes, aromas, pigmentos ou agentes antioxidantes e antimicrobianos (SAYURITANADA-PALMU et al., 2005).

Recobrimentos com gelatina, amido e gomas têm sido eficientes na conservação das propriedades dos alimentos. A gelatina é um produto à base de proteína e possui colágeno, importante para o consumo humano, mas sua eficácia na conservação de massa depende do produto a ser recoberto. A adição de alguns tipos de gomas, substâncias com atividade antimicrobiana e antioxidante como os óleos essenciais, entre outros aditivos, nos recobrimentos têm ajudado a melhorar as propriedades dos mesmos, como menor perda de massa, menor crescimento microbiano, além de manter a cor e a firmeza dos alimentos (RICARDO et al., 2014).

O objetivo do presente estudo foi avaliar o efeito da cobertura comestível biodegradável de gelatina adicionada de óleo essencial de alecrim nas características físicoquímicas de cortes de carne suína refrigerado.

\section{MATERIAL E MÉTODOS}

O desenvolvimento do trabalho foi realizado no Laboratório de Processamento de Alimentos da Universidade Federal de Viçosa/Campus Rio Paranaíba. Para aplicação dos revestimentos nos cortes suínos, utilizou-se a técnica de imersão dos cortes na solução do revestimento em temperatura ambiente $\left(\sim 25^{\circ} \mathrm{C}\right)$ para que ocorresse a polimerização do revestimento nos cortes. 


\section{The Journal of Engineering and Exact Sciences - \\ JCEC \\ ISSN: 2527-1075}

Os cortes, de aproximadamente $100 \mathrm{~g}$, foram imersos nas soluções de revestimento durante $5 \mathrm{~s}$, posteriormente, foram pendurados através de ganchos em câmara climática do tipo BOD, com temperatura de $4{ }^{\circ} \mathrm{C}$, durante $30 \mathrm{~min}$, até a completa secagem do revestimento.

Foram gerados 4 tipos de revestimento: CRX: cortes revestidos com cobertura de gelatina adicionada de 0,4 mg.L-1 de óleo essencial de alecrim; CRY: cortes revestidos com cobertura de gelatina adicionada de $0,8 \mathrm{mg} . \mathrm{L}-1$ de óleo essencial de alecrim; CR: cortes revestidos com cobertura de gelatina sem adição de óleo essencial de alecrim; e SR: controle, cortes sem recobrimento algum.

Os cortes foram acondicionados em bandejas de poliestireno e envoltos por filme plástico de PVC. Os tratamentos devidamente acondicionados, foram armazenados em câmara climática tipo BOD, à temperatura controlada de $4 \pm 0,5^{\circ} \mathrm{C}$, e foram analisados com 0 , 2, 4, 6 e 8 dias de armazenamento.

As análises físico-químicas de $\mathrm{pH}$, umidade e cinzas seguiram as metodologias descritas pelo Instituto Adolfo Lutz (2008). A perda de peso foi calculada pela diferença entre o peso final e inicial das amostras após os dias de armazenamento. A firmeza foi analisada a partir da força necessária para romper o tecido muscular, utilizando um penetrômetro manual, modelo PTR-300, marca Instrutherm, com ponteira de $5 \mathrm{~mm}$ de diâmetro.

\section{RESULTADOS E DISCUSSÃO}

Houve efeito significativo da interação tipo de revestimento $\mathrm{x}$ tempo de armazenamento para o $\mathrm{pH}$. Nota-se que durante todo o armazenamento, o $\mathrm{pH}$ dos cortes suínos em todos as amostras aumentaram (Figura 1). De acordo com Normam (2003), o pH encontrado inicialmente nos cortes suínos apresenta-se normal, sabendo que o tempo de início é caracterizado pelo evento de post mortem, no qual o $\mathrm{pH}$ muscular no abate se encontra em torno de 7,2, decresce para valores próximos de 6,0 e estabiliza em valores aproximados de 5,6, como pode ser observado na Figura 1.

Sales et al. (2013), encontrou valores próximos que variavam de 5,4 a 6,38 em carne suína in natura. Segundo o Ministério da Agricultura, carnes frescas de suínos que apresentam pH acima de 6,4 estão em estágio de degradação, mostrando que no último dia de armazenamento, os cortes suínos apresentavam indícios de degradação. 


\section{The Journal of Engineering and Exact Sciences - \\ JCEC}

ISSN: 2527-1075

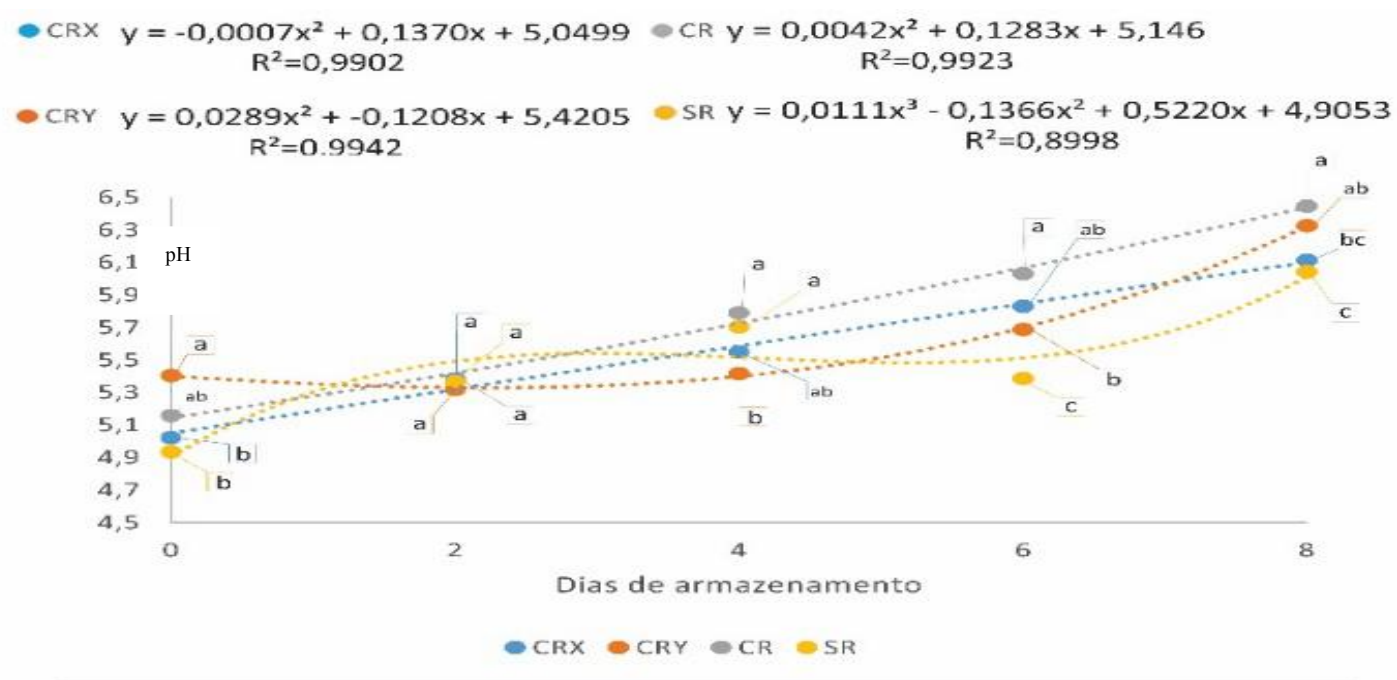

Figura 1 - Valores de $\mathrm{pH}$ de cortes de lombo suíno durante 8 dias de armazenamento. Valores com letras diferentes apresentaram diferença significativa $(p<0,05)$ pelo teste de Tukey. CRX: cortes revestidos com cobertura de gelatina adicionada de $0,4 \mathrm{mg} . \mathrm{L}-1$ de óleo essencial de alecrim; CRY: cortes revestidos com cobertura de gelatina adicionada de 0,8 mg.L-1 de óleo essencial de alecrim; CR: cortes revestidos com cobertura de gelatina; e SR: controle.

A umidade foi afetada significativamente pelo tipo de revestimento, perda de peso e firmeza foram afetadas pelo tipo de revestimento e tempo de armazenamento separadamente e cinzas não houve efeito significativo de nenhum dos fatores. As médias gerais dos tratamentos estão apresentadas na Tabela 1 , juntamente com teste Tukey ( $p>0,05)$.

A umidade da carne está diretamente relacionada com a sua suculência, sendo um fator fundamental para sua aceitação no mercado (RODRIGUES, 2004). Os valores encontrados nas análises para umidade ficaram na média de $72 \%$ para todos os tratamentos. Torres et al. (2000) em sua pesquisa com carne bovina, encontrou teores de umidade em torno de $74 \%$.

Tabela 1 - Médias dos valores de umidade e firmeza de cortes de carne suína armazenados sob refrigeração por 8 dias

\begin{tabular}{ccc}
\hline Tratamento & Umidade (\%) & Firmeza (N) \\
\hline CRX & $73,32 \pm 0,76 \mathrm{a}$ & $6,86 \pm 0,61 \mathrm{a}$ \\
CRY & $72,68 \pm 1,22 \mathrm{a}$ & $6,71 \pm 0,43 \mathrm{a}$ \\
CR & $72,75 \pm 0,24 \mathrm{ab}$ & $6,96 \pm 0,66 \mathrm{a}$ \\
SR & $71,73 \pm 0,91 \mathrm{~b}$ & $6,4 \pm 0,56 \mathrm{~b}$
\end{tabular}

Valores com letras diferentes apresentam diferença significativa $(p<0,05)$. CRX: cortes revestidos com cobertura de gelatina adicionada de 0,4 mg.L-1 de óleo essencial de alecrim; CRY: cortes revestidos com cobertura de gelatina adicionada de 0,8 mg.L-1 de óleo essencial de alecrim; CR: cortes revestidos com cobertura de gelatina; e SR: controle. 


\section{The Journal of Engineering and Exact Sciences - \\ JCEC}

ISSN: 2527-1075

A menor perda de peso observada nos tratamentos CRX, CRY e CR ocorre devido à presença do filme, pois a gelatina quando usada para a produção de biopolímeros confere característica de barreira a gases e vapores d'água. (PEREDA et al., 2011). O teor de cinzas para os tipos de revestimento não apresentou diferença significativa, tendo uma média de $1,29 \%$ de cinzas.

$\mathrm{Na}$ figura 2, estão representados os resultados dos tipos de revestimentos para perda de peso e firmeza em cada tempo de análise. Observa-se que houve perda de peso e da firmeza ao longo do armazenamento. De acordo com Oetterer (2008), a presença de enzimas proteolíticas ocasiona na degradação da actomiosina, ocorrendo o amolecimento da carne. E também, essas modificações quanto à perda de massa e ao amolecimento, pode ser devido a carne estar no início da degradação, como constatado na análise de $\mathrm{pH}$.

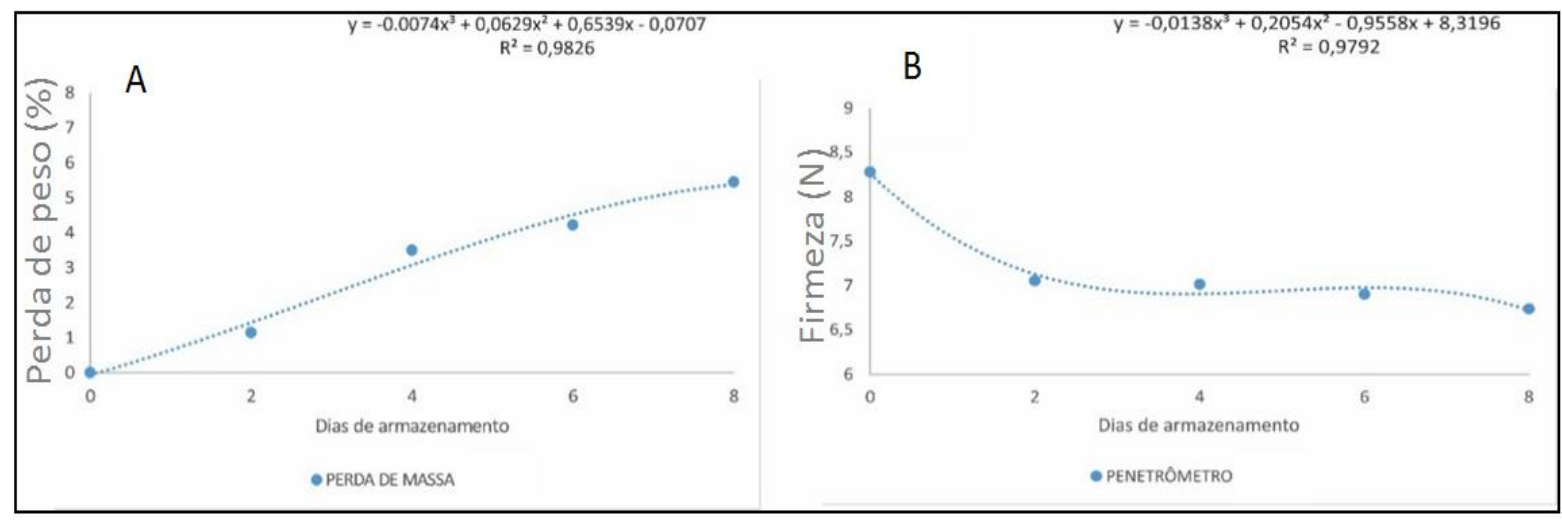

Figura 2 - Valores médios de perda de peso (A) e de firmeza (B) de cortes de lombo suíno armazenados por 8 dias.

\section{CONCLUSÕES}

O revestimento de gelatina adicionado de óleo essencial de alecrim ou não é eficiente para preservação da umidade, da perda de peso e da firmeza de cortes de lombo suíno, podendo assim ser utilizado como um tipo de embalagem primária para cortes de carne, já que o revestimento atua na manutenção das características importantes da carne suína. É necessário aprofundar este estudo para podermos avaliar o efeito dos revestimentos quanto à conservação microbiológica.

\section{REFERÊNCIAS}

AOAC - Association of Official Analytical Chemists. HORWITZ, W. Official methods of analysis of the Association of Ofificial Analytical Chemists. 17ed. Arlington: AOAC Inc., v1 e v2, 2000.

BRASIL. Ministério da Agricultura e do Abastecimento. Métodos Analíticos para Controle de Produtos de Origem Animal e seus Ingredientes - LANARA. Brasília, 1989.

CARDOSO, G. P. Revestimento comestível para comercialização de carne bovina refrigerada. 2015. 129f. Tese (Doutorado em Ciências dos Alimentos). Universidade Federal de Lavras, Lavras. 2015. 
FAO. Food and Agriculture organization and the United Nations. Production / Live Animals. 2016. Disponível em: <http://faostat3.fao.org/browse/Q/QA/E >. Acesso em: 18/04/2016.

INSTITUTO ADOLFO LUTZ - IAL. Métodos Físico-Químicos para Análise de Alimentos. 4.ed. 1.ed. digital. São Paulo: IAL, 2008.

MAPA. Ministério da Agricultura, Pecuária e Abastecimento. Suínos. 2016. Disponível em: $<$ http://www.agricultura .gov.br/animal/especies/suinos>. Acesso em: 18/04/2016.

NORMAN, J.L. et al. Pork loin color relative to sensory and instrumental tenderness and consumer acceptance. Meat Science, v. 65, p. 927-933, 2003.

OETTERER, M. Tecnologia de Pescado. Universidade de São Paulo. Escola Superior de Agricultura "Luiz de Queiroz". Departamento de Agroindústria, Alimentos e Nutrição.

PEREDA, M. et al. Chitosan-gelatin composites and bi-layer films with potential antimicrobial activity. Food Hydrocolloids, Oxford, v. 25, n. 5, p. 1372-1381, June 2011.

RICARDO, L. P.; MORAIS, M. M.; ROSA, G. S. da. Estudo de filmes biodegradáveis de recobrimentos aplicado em morangos. XX Congresso Brasileiro de Engenharia Química. Florianópolis, Santa Catarina, 2014.

RODRIGUES, V.C.; BRESSAN, M.C.; CARDOSO, M.G.; FREITAS, RTF. Ácidos graxos na carne de búfalos e bovinos castrados e inteiros. R. Bras. Zoot. 2004.

SALES. L. E. M. et al. Avaliação da carne suína in natura comercializada em Mossoró/ RN. Acta Veterinaria Brasilica, v.7, n.4, p.306-310, 2013

SARCINELLI, M. F.; VENTURINI, K. S.; SILVA, L. C. da. Processamento de carne suína. Boletim técnico, Universidade Federal do Espírito Santo, 2007.

SAYURITANADA-PALMU, P.; PROENÇA, P. de S. P.; TRANI, P. E.; PASSOS, F. A.; GROSSO, C. R. F. Recobrimento de sementes de brócolis e salsa com coberturas de filmes biodegradáveis. Bragantia, Campinas, São Paulo, v. 64, n. 2, p. 391-397, 2005.

TORRES, E. A. F. S.; CAMPOS, N. C.; DUARTE, M.; GARBELOTTI, M. L.; PHILIPPI, S. T.; RODRIGUES, S. M. Composição centesimal e valor calórico de alimentos de origem animal. Ciênc. Tecnol. Aliment. 2000.

ZAMBIAZI, R. C. Análise físico-química de alimentos. Pelotas: Editora Universitária/UFPEL. 202 p. 2010. 